\begin{tabular}{|c|l|}
\hline Title & Catalyst-free growth of GaA s nanowires by selective area metal organic vapor-phase epitaxy \\
\hline Author(s) & Noborisaka, Jinichiro; Motohisa, Junichi; Fukui, Takashi \\
\hline Citation & $\begin{array}{l}\text { A pplied Physics Letters, 86(21), 213102 } \\
\text { https://doi.org/10.1063/1935038 }\end{array}$ \\
\hline Issue Date & 2005-05-23 \\
\hline Doc URL & http://hdl.handle.net/2115/5506 \\
\hline Rights & Copyright $\odot 2005$ A merican Institute of Physics \\
\hline Type & article \\
\hline File Information & APL86-21.pdf \\
\hline
\end{tabular}

Instructions for use 


\title{
Catalyst-free growth of GaAs nanowires by selective-area metalorganic vapor-phase epitaxy
}

\author{
Jinichiro Noborisaka, ${ }^{a)}$ Junichi Motohisa, and Takashi Fukui \\ Graduate School of Information Science and Technology and Research Center for Integrated Quantum \\ Electronics (RCIQE), Hokkaido University, North 13, West 9, Sapporo 060-8628, Japan
}

(Received 7 June 2004; accepted 9 April 2005; published online 16 May 2005)

\begin{abstract}
We report on the fabrication of GaAs hexagonal nanowires surrounded by $\{110\}$ vertical facets on a GaAs (111) B substrate using selective-area (SA) metalorganic vapor-phase epitaxial (MOVPE) growth. The substrate for $\mathrm{SA}$ growth was partially covered with thin $\mathrm{SiO}_{2}$, and a circular mask opening with a diameter $d_{0}$ of 50-200 nm was defined. After SA-MOVPE, GaAs nanowires with a typical diameter $d$ ranging from 50 to $200 \mathrm{~nm}$ and a height from 2 to $9 \mu \mathrm{m}$ were formed vertically on the substrate without any catalysts. The size of the nanowire depends on the growth conditions and the opening size of the masked substrate. A possible growth mechanism is also discussed. (C) 2005 American Institute of Physics. [DOI: 10.1063/1.1935038]
\end{abstract}

Nanostructures, such as nanotubes, nanowires, and quantum dots, offer possibilities for achieving revolutionary advances toward nanotechnology-based electronics, optoelectronics, and biotechnology. For example, semiconductor nanowires, made from materials such as $\mathrm{Si}, \mathrm{Ge}, \mathrm{GaP}, \mathrm{GaAs}$, and InP, have been studied for their application in logic circuits, ${ }^{1}$ ultrasmall light emitters, ${ }^{2-4}$ and nanoscale photodetectors. ${ }^{5}$ So far, these semiconductor nanowires have been fabricated by a vapor-liquid-solid (VLS) growth method using metal catalysts. ${ }^{6}$

In our previous study, we proposed an approach for forming semiconductor nanowires by using selective-area metalorganic vapor phase epitaxy (SA-MOVPE). ${ }^{7}$ This was inspired by SA-MOVPE growth of GaAs (Ref. 8) or InGaAs (Ref. 9) pillar arrays on an (111)B-oriented surface for applications of photonic crystals. A similar SA-MOVPE method for InP nanowire is also reported by Poole et al. ${ }^{10} \mathrm{By}$ using SA-MOVPE, we can fabricate nanowires at predetermined positions without the help of catalysts and we can adopt atomically precise controllability of epitaxial growth. It is possible, therefore, to achieve heterostructures with abrupt heterojunctions in the growth direction (vertical heterostructures). Furthermore, lateral growth, as well as vertical growth, can be controlled independently by appropriate growth conditions to passivate the surfaces of wires. Previously reported semiconductor nanowires formed by VLS growth are mostly insulating with high-density surface states. Surface passivation and lateral heterostructures are, therefore, extremely important for practical applications of nanowires as well as for optical and transport measurements.

In this study, we report on the growth of very thin GaAs nanowires with SA-MOVPE. We grew hexagonal nanowires with a diameter $d$ of 50-200 $\mathrm{nm}$. The growth mechanism of nanowires is discussed, based on the diameter $d$ and the pattern dependency of the height $h$ of the nanowires.

Figure 1(a) shows schematic illustrations of the fabrication process of nanowires. This fabrication process is essentially the same as the one for GaAs, ${ }^{8}$ InGaAs, ${ }^{9}$ and InP (Ref. 11) pillars on masked substrates except for the size of the mask opening. After $20 \mathrm{~nm}$ thick $\mathrm{SiO}_{2}$ was deposited by

\footnotetext{
a) Author to whom correspondence should be addressed; electronic mail: nobori@rciqe.hokudai.ac.jp
}

plasma sputtering on GaAs (111) B substrates, periodic hexagonal opening patterns were formed by electron-beam (EB) lithography, and by wet-chemical etching techniques. Hexagonal openings were arranged in a triangular lattice [see Fig. 1(b)], with the pitch $a$ ranging from 0.4 to $3 \mu \mathrm{m}$. The actual shape of the opening tended to be circular because of the resolution limit in EB lithography and wet chemical etching. As we later describe, the diameter $d$ of nanowires is directly related to the opening diameter $d_{0}$, and a smaller $d_{0}$ results in a longer nanowire height $h$. Therefore, we tried to obtain the smallest $d_{0}$ possible by controlling the amount of the EB dose. For this reason, the size of the opening area sometimes became nonuniform in the minimal EB dose conditions [see Fig. 1(b)]. The diameter $d_{0}$ of the opening area was changed from 50 to $200 \mathrm{~nm}$. This periodic array of the opening pattern was only defined in $100 \times 100 \mu \mathrm{m}$ square regions, and outside of the square regions $\mathrm{SiO}_{2}$ was removed and the GaAs surface was exposed.

GaAs nanowire growth was carried out in a horizontal MOVPE system working at 0.1 atm. Trimethylgallium (TMG) and $20 \%$ arsine $\left(\mathrm{AsH}_{3}\right)$ diluted in $\mathrm{H}_{2}$ were used as source materials. The partial pressure of TMG was 2.7 $\times 10^{-7}$ atm and that of $\mathrm{AsH}_{3}$ was changed from $2.5 \times 10^{-4}$ to $1.7 \times 10^{-3} \mathrm{~atm}$. The growth temperature was $750{ }^{\circ} \mathrm{C}$. In these conditions, the growth rate of GaAs on a planar (001) substrate is $2.1 \AA / \mathrm{s}$ and $0.3 \AA / \mathrm{s}$ on (111) B, but the growth rate of nanowires is much higher, as will be discussed later. The total growth time was $20 \mathrm{~min}$.

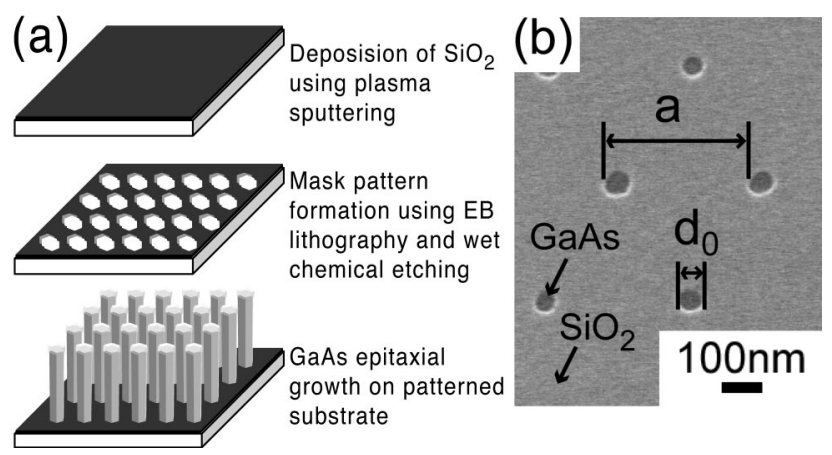

FIG. 1. (a) Schematic illustration of the fabrication process. (b) SEM image of the mask pattern on a GaAs (111) B substrate. The pitch $a$ of the triangular lattice is $0.5 \mu \mathrm{m}$ for this pattern. 




FIG. 2. SEM images of GaAs nanowires for pattern opening $d_{0}$ of (a) 200 $\mathrm{nm}$ and (b) $50 \mathrm{~nm}$. The inset in (b) is an image from the top.

Figures 2(a) and 2(b) show secondary electron microscopy (SEM) images for two typical samples of GaAs nanowires grown on a GaAs (111) B masked substrate at an $\mathrm{AsH}_{3}$ partial pressure of $5.0 \times 10^{-4} \mathrm{~atm}$. The pattern period $a$ is $1 \mu \mathrm{m}$, and the diameter $d_{0}$ is $200 \mathrm{~nm}$ and $50 \mathrm{~nm}$ for samples Fig. 2(a) and 2(b), respectively. GaAs nanowires were grown in a (111) B (vertical) direction on the substrate. As shown in the inset in Fig. 2(b), the nanowires have a hexagonal cross section. From the symmetry, we conclude that the nanowires have six $\{110\}$ side facets similar to the larger hexagonal structure reported previously. ${ }^{12}$ The diameter $d$ of the nanowires becomes smaller as the initial opening diameter $d_{0}$ is reduced. For sample Fig. 2(a), the diameter $d$ and the height $h$ of the nanowires are $200 \mathrm{~nm}$ and $2.8 \mu \mathrm{m}$, while for sample Fig. 2(b), these are $60 \mathrm{~nm}$ and $5 \mu \mathrm{m}$, respectively. Furthermore, for the latter, nonuniform growth was observed. We believe that this originated mainly from the nonuniform mask opening size, as observed in Fig. 1(b). As far as the growth mechanism is concerned, this nonuniformity does not alter our main discussions.

Figure 3(a) shows the height $h$ of GaAs nanowires versus nanowire diameter $d$. The pattern period $a$ is $0.6 \mu \mathrm{m}$. The height $h$ is much higher than the typical growth thickness on a planar (111) B GaAs substrate (42 nm), which is indicated by the horizontal line in the figure. Furthermore, $h$ increases as $d$ decreases. It is also noted that $h$ also increases as $\left[\mathrm{AsH}_{3}\right]$ decreases. These results are consistent with the previous results for SA-MOVPE of GaAs hexagonal structures. ${ }^{8} h$, however, also increases as the pitch $a$ of the opening decreases, as shown in Fig. 3(b). We obtained the narrowest and longest nanowires with $50 \mathrm{~nm}$ and $9 \mu \mathrm{m}$, achieving an aspect ratio of 180 at $\left[\mathrm{AsH}_{3}\right]=2.5 \times 10^{-4} \mathrm{~atm}$.

Within the growth conditions we used, where the growth temperature was relatively high and $\left[\mathrm{AsH}_{3}\right]$ was low, as compared to the ordinary GaAs growth conditions, we expected that the growth rate on (111) B would be much higher than that on a (110) surface. ${ }^{12,13}$ This is because adsorbed Ga atoms do not have strong bonding on a (110) surface for small As coverages, and they can desorb very easily. In fact, according to our SEM study, almost no lateral growth over the $\mathrm{SiO}_{2}$ mask was observed, indicating that there is almost no growth on $\{110\}$ vertical surfaces and thus $d \sim d_{0}$. Formation of hexagonal structures, therefore, was explained by the six-fold symmetry of the (111) B-oriented surface and the fact that $\{110\}$ vertical facets exhibited the slowest growth rate. The $\mathrm{AsH}_{3}$ partial pressure dependence on the nanowire height $h$ could qualitatively be explained by the surface reconstruction of (111) B GaAs surfaces. ${ }^{14}$ That is, when the $\mathrm{AsH}_{3}$ partial pressure is high, the growth of the (111) B surface is suppressed by the formation of stable As trimers, Downloaded 23 Jan 2006 to 133.87.26.100. Redistribution subject
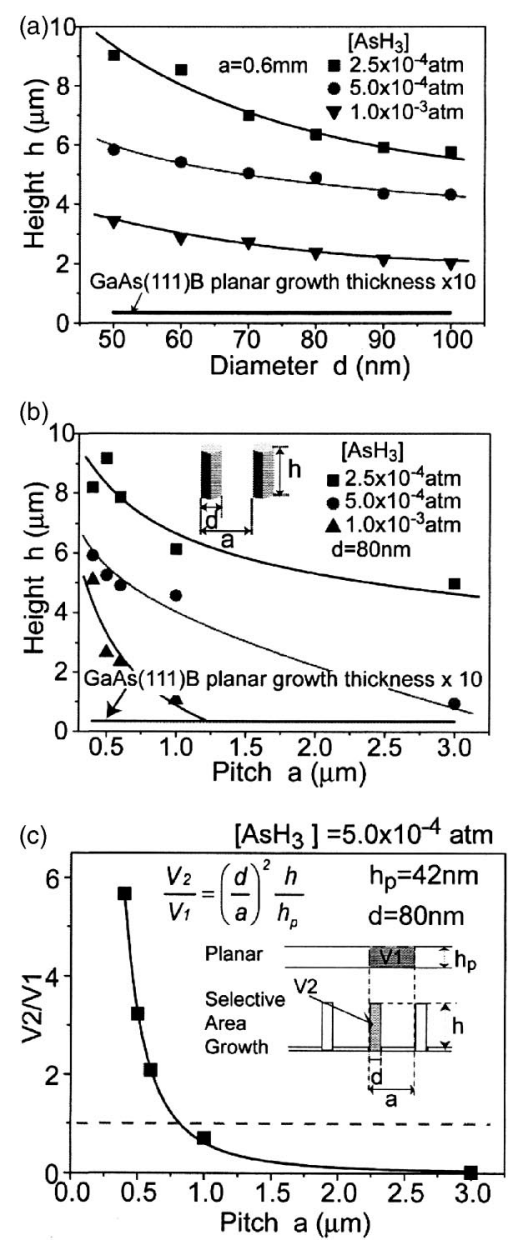

FIG. 3. Height $h$ of GaAs nanowires versus (a) nanowire diameter $d$ and (b) pattern pitch $a$. (c) Volume ratio $V_{2} / V_{1}$ of GaAs grown on patterned and planar substrates in a unit cell of the triangular lattice. Growth volume $V_{l}$ is for the planar and $V_{2}$ is for the nanowires, as shown in the inset.

whereas they become less stable at lower partial pressure, resulting in an increase in the growth rate. However, the enhancement of the growth rate on patterned substrates and the dependence of $h$ on the pattern geometry $\left(a\right.$ and $\left.d_{0}\right)$ cannot be explained in the simple model.

To examine the growth rate enhancement in more detail, we plot in Fig. 3(c) the calculated total volume $V_{2}$ of the nanowires in a unit cell of the triangular lattice on a patterned substrate normalized by the volume $V_{1}$ of a planar growth in the same area. The area of the unit cell is given by $a^{2}$ and the cross-sectional area of hexagonal nanowires is given by $d^{2}$. The ratio $V_{2} / V_{1}$ is therefore calculated as $(d / a)^{2}\left(h / h_{p}\right)$, where $h_{p}$ is the growth thickness on a planar substrate and is $42 \mathrm{~nm}$. We can see a significant increase in $V_{2} / V_{1}$ as $a$ becomes smaller. Note that $V_{2} / V_{1}$ should go to 1.0 in the limit $a \rightarrow 0$, though it looks unlikely at first sight within the present experimental results.

In the case of SA-MOVPE on a (100) GaAs surface, the enhancement of the growth rate in the opening region of the mask is generally observed. This is explained by the gasphase and/or surface diffusion of the growth species from the masked to opening regions. This enhancement is known to be dependent on the pattern geometry, and in the present case where the patterned area is limited to $100 \times 100 \mu \mathrm{m}$ regions, we showed that the enhancement was only about 20 to $30 \%$ of the planar growth rate. ${ }^{15,16}$ If we take into account the fraction $f$ of the mask opening [in the present case, $\left(d_{0} / a\right)^{2}$ ] 
in the patterned regions, the growth rate increases slightly as $f$ is reduced, as expected from the diffusion of growth species. Nevertheless, the ratio $V_{2} / V_{1}$ of the total growth amount between the patterned and planar growth does not exceed unity as reported in Ref. 17. That is, the total amount of growth is always smaller on patterned substrates than on planar substrates, indicating that the thermal decomposition of the column III precursor is effectively decreased in the patterned area or a considerable amount of the growth species diffuse out to the exposed regions surrounding the patterned regions. This simple gas-phase/surface diffusion model can partly explain our present results. As in Fig. 3(a), $h$ increases as $f$ decreases. This simple model, however, completely contradicts the results of Figs. 3(b) and 3(c).

To get more insight for the growth process of the nanowires, we considered diffusion processes in and between three different regions, namely, (111) B top surface, $\{110\}$ sidewall facets, and masked areas. In this model, we assume the following diffusion equation for the concentration $N$ of growth species on the surface in each region and that the growth proceeds by the incorporation of species into the crystal phase:

$$
\frac{d}{d x}\left(D \frac{d N}{d x}\right)=J+\frac{N}{\tau}
$$

Here, $D$ is the surface diffusion constant, $1 / \tau=1 / \tau^{\prime}+1 / \tau^{\prime \prime}$, where $\tau^{\prime}$ is the incorporation time, $\tau^{\prime \prime}$ is the re-evaporation time of species, and $J$ is the impinging flux of growth species from the vapor phase. It is also assumed that a complicated interplay of gas-phase diffusion can be considered within effective flux $J$. All of these parameters are defined in each of the three regions [we use subscript (111)B, $\{110\}$, and $m$ for their distinction], and the continuity of the flux at the boundary of different regions is used as the boundary condition. Then, the growth rate is proportional to $N \tau^{\prime}$. The conclusion of our model is that the dominant factor of diffusion between different regions is $J \tau$, which is exactly the surface concentration of the growth species and is readily acceptable if one thinks of the nature of diffusion. Thus, if three regions considered here take a different $J \tau$, there could be diffusion between (111)B and $\{110\}$ surfaces, $\{110\}$ surfaces or masked regions, as usually seen in SA-MOVPE.

If we assume $d \ll L_{(111) B}$, where $L_{(111) B}=\sqrt{D_{(111) B} \tau_{(111) B}}$ is the diffusion length and is thought to be in the order of a few microns, and no incorporation into the crystal phase in $\{110\}$ and masked region (that is, $\tau_{\{110\}}^{\prime}$ and $\tau_{m}^{\prime}$ are infinite), we find that the most relevant parameters are $J_{\{110\}} \tau_{\{110\}}^{\prime \prime}$, $J_{\mathrm{m}} \tau_{m}^{\prime \prime}$ and $J_{\{110\}} \tau_{\{110\}}^{\prime \prime}<J_{m} \tau_{m}^{\prime \prime}$. Note here that, if these parameters are independent of the period $a$ of the mask pattern and size $d$ and $h$ of nanowires, we end up with the conventional model of SA-MOVPE mentioned earlier, and we cannot explain the anomalous enhancement of the growth rate and diffusion.

Thus, to explain the experimental data, we have to think $J_{\{110\}} \tau_{\{110\}}^{\prime \prime}$ to be increased, or $J_{m} \tau_{m}$ to be decreased as $a$ is decreased: In fact, there are several possibilities. If the species once desorbed from the masked area are readsorbed on $\{110\}$ surfaces, particularly, in dense pillar arrays, this gives additional contribution to $J_{\{110\}}$. It is also possible that $\tau_{m}$ is effectively shortened when $a$ is reduced, since the species diffusing or migrating on the mask can easily find the nanowires to be incorporated. At this moment, we are not able to convince ourselves what kind of processes is possible and important, and clarification of the anomalous diffusion is left for future study.

Finally, we discuss our various approaches for semiconductor nanowires. We have already reported on hexagonal pillar structures of InGaAs on (111) B InP, InP on (111) A InP with a relatively large size $d(\sim 100 \mathrm{~nm})$. Miniaturization of the nanowire diameter around $50 \mathrm{~nm}$ is thought to be possible simply by reducing the size of the mask opening. In fact, we have already obtained InGaAs nanowires with $d$ $\sim 60 \mathrm{~nm}$ and $h \sim 5.6 \mu \mathrm{m}$ by SA-MOVPE on (111)B InP substrates. We have also demonstrated incorporating $\mathrm{Al}$ into GaAs-based nanowires. Furthermore, for SA-MOVPE on (111) B GaAs, the growth on $\{110\}$ sidewalls is possible through control of growth conditions. ${ }^{18}$ These lead us to achieve nanowires with heterojunctions independently in both vertical and lateral directions to form core shell or other complicated structures. We shall describe elsewhere a detailed study on the growth mechanism of these nanowire vertical lateral heterostructures.

The authors acknowledge Professor H. Hasegawa, Professor C. Tu, Dr. S. de Franceschi, and Dr. M. Akabori for helpful discussions, and J. Takeda, Y. Miyoshi, N. Ooike, M. Inari, A. Koike, and S. Akamatsu for experimental support. This work was financially supported in part by the Grant-inAid for Scientific Research, and by the Japan Society of Promotion of Science.

${ }^{1}$ Y. Huang, X. Duan, Y. Cui, L. J. Lauhon, K. Kim, and C. M. Lieber, Science 294, 1313 (2001).

${ }^{2}$ M. H. Huang, S. Mao, H. Feick, H. Yan, Y. Wu, H. Kind, E. Weber, R. Russo, and P. Yang, Science 292, 1897 (2001).

${ }^{3}$ N. Panev, A. I. Persson, N. Skold, and L. Samuelson, Appl. Phys. Lett. 83, 2238 (2003).

${ }^{4}$ X. Duan, Y. Huang, R. Agarwal, and C. M. Lieber, Nature (London) 421, 241 (2003).

${ }^{5}$ J. Wang, M. S. Gudiksen, X. Duan, Y. Cui, and C. M. Lieber, Science 293, 1455 (2001).

${ }^{6}$ K. Hiruma, H. Yazawa, T. Katuyama, K. Ogawa, K. Haraguchi, M. Koguchi, and H. Kakibayhasi, J. Appl. Phys. 77, 447 (1995).

${ }^{7}$ J. Motohisa, J. Takeda, M. Inari, J. Noborisaka, and T. Fukui, Physica E (Amsterdam) 23, 298 (2004).

${ }^{8}$ T. Hamano, H. Hirayama, and Y. Aoyagi, Jpn. J. Appl. Phys., Part 2 36, L286 (1997)

${ }^{9}$ M. Akabori, J. Takeda, J. Motohisa, and T. Fukui, Nanotechnology 14, 1071 (2003).

${ }^{10}$ P. J. Poole, J. Lefebvre, andJ. Fraser, Appl. Phys. Lett. 83, 2055 (2003).

${ }^{11}$ M. Inari, J. Takeda, J. Motohisa, and T. Fukui, Physica E (Amsterdam) 21, 620 (2004).

${ }^{12}$ S. Ando, N. Kobayashi, and H. Ando, J. Cryst. Growth 145, 302 (1994).

${ }^{13}$ T. Fukui, S. Ando, and Y. Tokura, Appl. Phys. Lett. 58, 2018 (1991).

${ }^{14}$ T. Hayakawa and M. Morishima, Appl. Phys. Lett. 59, 3321 (1991).

${ }^{15}$ K. Kumakura, K. Nakakoshi, M. Kishida, J. Motohisa, T. Fukui, and H. Hasegawa, J. Cryst. Growth 145, 308 (1994).

${ }^{16}$ Y. Aritsuka, T. Umeda, J. Motohisa, and T. Fukui, Mater. Sci. Proc. 570, 97 (1999).

${ }^{17}$ S. Ando, Ph.D. thesis (1998, in Japanese).

${ }^{18}$ S. Ando, N. Koybayashi, and H. Ando, Jpn. J. Appl. Phys., Part 2 37, L105 (1998). 